\title{
CoMFA and CoMSIA on the Inhibition of Calcineurin-NFAT Signaling by Blocking Protein-Protein Interaction with $\mathrm{N}$-(4-Oxo-1(4H)-naphthalenylidene)benzenesulfonamide Derivatives
}

\author{
Pyung-Keun Myung, Kyung-Yong Park, ${ }^{\dagger}$ and Nack-Do Sung ${ }^{\ddagger}, *$ \\ College of Pharmacy, Chungnam National University, Daejeon 305-764, Korea \\ ${ }^{\dagger}$ Research Center for Transgenic Cloned Pigs, Chungnam National University, Daejeon 305-764, Korea \\ ${ }^{\star}$ Division of Applied Biology \& Chemistry, Chungnam National University, Daejeon 305-764, Korea \\ *E-mail: ndsung@cnu.ac.kr \\ Received June 22, 2005
}

\begin{abstract}
To raises the possibility of designing effective inhibitors, 3D-QSAR for the inhibition of calcineurin-NFAT signaling by new $\mathrm{N}$-(4-oxo-1(4H)-naphthalenylidene)benzenesulfonamide derivatives as inhibitors of intracellular protein-protein interactions were studied using CoMFA and CoMSIA methodology. The three templates, $N$-(4-oxo-1(4H)-naphthalenylidene)benzenesulfonamide (A), benzenesulfonamide (B) and 4-oxo$1(4 \mathrm{H})$-naphthalenylidene $(\mathrm{C})$ were selected to improve the statistic of the present 3D-QSAR models. The best models with combination of standard field in CoMFA, and steric field and electrostatic field in CoMSIA derived from the template, $\mathrm{B}$ and $\mathrm{C}$, because most of the compounds tend not to be aligned in template $\mathrm{A}$. From the based on the CoMFA and CoMSIA contour maps, the $\mathrm{R}_{1}$ and $\mathrm{R}_{2}$ groups on 4-oxo-1(4H)-naphthalenylidene ring are steric favor. The ortho position on the benzenesulfonyl ring is steric disfavor and the meta position is steric favor. In addition, the oxygene atom of carbonyl group will have better inhibition activities as it has a negative charge favor. From these findings, we can conclude that the analyses of the contour maps provided insight into possible modification of molecules for effective inhibitiors.
\end{abstract}

Key Words : $N$-(4-Oxo-1(4H)-naphthalenylidene)benzenesulfonamides, Immunomodulatory agent, Calcineurin-NFAT signaling, CoMFA \& CoMSIA model

\section{Introduction}

The modern era of transplantation began with the emergence of a potent immunosuppressive drug, cyclosporine (CsA), FK506 and rapamycin. The proteins targeted by these drugs include the binding proteins named immunophilins and the drug targets calcineurin $(\mathrm{CN})$ and target of rapamycin. The ubiquity of these proteins raises the question of why these drugs affect the immune response relatively and selectively. ${ }^{1} \mathrm{CN}$ is a ubiquitous enzyme that plays a role in a number of cellular processes. Cell signaling through phosphatase and kinase cascades is mediated by protein : protein interactions via scaffolding, anchoring and adapter proteins. $^{2} \mathrm{CN}$ has been shown to directly modulate the activity of a number of intracellular proteins. Some are transcription factors, such as nuclear factor of activated $\mathrm{T}$ cells (NFAT), while others are enzymes, such as nitric oxide synthase.

The members of the NFAT family of transcription factors are the most well-characterized substrate of CN. NFAT is found in its phosphorylated state in the cytosol in resting cells. Upon activation by an increase in intracellular $\mathrm{Ca}^{++}$, $\mathrm{CN}$ binds to and dephosphorylates NFAT. ${ }^{3}$ Dephosphorylations of NFAT is hypothesized to unmask a nuclear localization sequence, resulting in the nuclear translocation of the NFAT-CN complex. ${ }^{4}$ Because of the ubiquitous expression and multiple substrates of $\mathrm{CN}$, more specific targeting of $\mathrm{CN}$ activity is required. NFAT peptides (SPRIETT or VIVIT) have been shown to be more pharmacologically specific in inhibiting the phosphatase activity of CN. The amino acid sequences of NFAT peptides correspond to one region on NFAT where $\mathrm{CN}$ binds. NFAT peptides are thought to disrupt the interaction between NFAT proteins and $\mathrm{CN}$ by binding to $\mathrm{CN}$ at the region where NFAT needs to lock and subsequently inhibits $\mathrm{CN}$ dephosphorylation of NFAT.

The peptides did not interfere with $\mathrm{CN}$ phosphatase activity against other substrates, such as the PKA-phosphorylated $\mathrm{K} \mathrm{II} \alpha$ and ErK-2-phosphorylated I, ${ }^{5}$ and selectively inhibited expression of NFAT-dependent cytokine genes without affecting the expression of $\mathrm{CN}$-dependent but NFAT-independent cytokine genes. ${ }^{6}$ It was identified that small organic molecules specifically block targeting of the protein phosphotase calcineurin to its substrate NFAT and was showed that they are effective inhibitors of calcineurinNFAT signaling. ${ }^{7}$

Here, to design some effective inhibitors, 3D-QSAR (three dimensional quantitative structure-activity relationship $)^{8}$ studies for the inhibition of calcineurin-NFAT signaling pathway by new $N$-(4-oxo-1(4H)-naphthalenylidene)benzenesulfonamide derivatives as inhibitors of intracellular protein-protein interactions were performed using comparative molecular field analyses (CoMFA) ${ }^{9}$ and comparative molecular similarity indices analyses $(\text { CoMSIA })^{10}$ methodologies. 


\section{Materials and Methods}

Substrate Molecules and Inhibition Activity. The inhibition $\left(\mathrm{K}_{\mathrm{d}}\right)$ values of calcineurin-NFAT signaling by blocking protein-protein interaction with $\mathrm{N}-(4-0 x-1(4 \mathrm{H})-$ naphthalenylidene)benzenesulfonamide derivatives based on fluorescence polarization assays were obtained. The substrate molecules, N-(4-oxo-1(4H)-naphthalenylidene)benzenesulfonamide derivatives as the inhibitors of NFATcalcineurin association were selected from the reference. ${ }^{7}$ The molar concentration of $50 \%$ inhibition activity $\left(\mathrm{IC}_{50}\right)$ was obtained by performing experiments at different concentrations and $\mathrm{pI}_{50}$ was calculated from following equation.

$$
\mathrm{pI}_{50}=-\log \left(\mathrm{IC}_{50} / \mathrm{M} . \mathrm{Wt} \times 1000\right)
$$

Molecular Modeling and Alignment. CoMFA ${ }^{9,10}$ and partial least square (PLS) analysis have been done using Sybyl, the molecular modeling software of the Tripos Inc. (Version 7.0). ${ }^{11}$ First, the most stable conformer of the molecule with no substituents, is investigated by using the simulated annealing method. ${ }^{12}$ In the Tripos force field, the partial charge of a specific atom is assigned using the Gasteiger-Hückel charge, ${ }^{13}$ and then the most stable conformer is obtained. In order to superimpose training set molecules, the common part of the molecule, N-(4-oxo-1(4H)naphthalenylidene)-benzenesulfonamide (A), is subdivided into two basic parts benzenesulfonamide (B) and 4-oxo1(4H)-naphthalenylidene (C) (Fig. 1), and each of them is superimposed $^{14}$ in the three dimensional space by the atom based fit method, ${ }^{15}$ and then aligned by alignment rule. ${ }^{16}$ Using templates $\mathrm{B}$ and $\mathrm{C}$, the CoMFA and CoMSIA models are derived. ${ }^{17}$

To validate our models, 2-3 compounds which are not included in the training set are assigned as a test set and their biological activities are estimated from the PLS results. Similarity indices in CoMSIA are calculated using a Gaussian-type distance dependence. The attenuation factor $\alpha$ was initially set to 0.3 . To decide the appropriate value, attenuation factor was varied in a parameter study within the range 0.1-0.9 in steps of 0.1 , and subsequently similarity indices and $\mathrm{q}^{2}$ values were computed each time (Fig. 4).

CoMFA and CoMSIA-PLS Analyses. We have placed each molecule aligned in the three dimensional space into the three dimensional cubic lattice space, with grid space from 1.0 to $3.0 \AA$ and regularly arranged the carbon atom $\left(\mathrm{sp}^{3},+1\right.$ charge). Then, in order to explain the dispersion between the molecules and the electrostatic field by the Coulombic potential between probe atoms and the rest areas of molecules, we have calculated the steric field energy (Van der Waals potential) by using the Lennard-Jones potential. ${ }^{9}$

To enhance the correlation between models, we have tried to find models derived from the standard field in the CoMFA and from the electrostatic and the steric fields in the CoMSIA. We have calculated the correlation between the descriptors of the three dimensional structural characteristics of the compounds aligned in the three dimensional space and their inhibition activities by PLS. ${ }^{18}$ To determine the optimal number of components (ONC), we have performed the LOO (leave-one-out) cross-validated process ${ }^{19}$ and obtained the value of $\mathrm{q}^{2}$ (or $\mathrm{r}_{\mathrm{cv} \text {. }}^{2}$. The $\mathrm{r}^{2}$ ncv. value, conventional coefficient, is obtained by non cross-validated process. When the value of $r^{2}$ ncv, which means the fitness of models, is more than 0.90 , and $\mathrm{q}^{2}$ (or $\mathrm{r}_{\mathrm{cv} \text {. }}^{2}$ ), which is the measurement of predictability, is more than 0.5 , the derived models seem to have stability and reasonable predictability. At this moment, if the number of components increases, the value of $\mathrm{q}^{2}$ (or $\mathrm{r}^{2}$ cv. ) also increases. To avoid the over fitting of data points, a

Table 1. Observed inhibition (Obs.pI $\left.{ }_{50}\right)$ of calcineurin-NFAT signaling with $N$-(4-oxo-1 $(4 \mathrm{H})$-naphthalenylidene)benzenesulfonamides and predicted (Pred.) by 3D-QSAR models: Atom based fit alignment and different template (B \& C)

\begin{tabular}{|c|c|c|c|c|c|c|c|c|c|c|c|c|c|c|}
\hline \multirow{3}{*}{ No. - } & \multirow{2}{*}{\multicolumn{5}{|c|}{ Substituents }} & \multirow{3}{*}{$\begin{array}{l}\text { Obs. } \\
\text { pI }_{50}\end{array}$} & \multicolumn{4}{|c|}{ CoMFA } & \multicolumn{4}{|c|}{ CoMSIA } \\
\hline & & & & & & & \multicolumn{2}{|c|}{ B } & \multicolumn{2}{|c|}{$\mathrm{C}$} & \multicolumn{2}{|c|}{$\mathrm{B}$} & \multicolumn{2}{|c|}{$\mathrm{C}$} \\
\hline & $\mathrm{R}_{1}$ & $\mathrm{R}_{2}$ & $\mathrm{R}_{3}$ & $\mathrm{R}_{4}$ & $\mathrm{R}_{5}$ & & Pred. $^{a}$ & Dev. $^{b}$ & Pred. $^{a}$ & Dev. $^{b}$ & Pred. $^{a}$ & Dev. $^{b}$ & Pred. $^{a}$ & Dev. $^{b}$ \\
\hline 1 & $\mathrm{Cl}$ & $\mathrm{Cl}$ & - & $\mathrm{H}$ & $\mathrm{H}$ & 6.45 & 6.33 & 0.12 & 6.55 & -0.10 & 6.51 & -0.06 & 6.34 & 0.11 \\
\hline 2 & $\mathrm{Cl}$ & $\mathrm{Cl}$ & - & $\mathrm{H}$ & $\mathrm{Me}$ & 6.54 & 6.22 & 0.32 & 6.44 & 0.10 & 6.41 & 0.13 & 6.43 & 0.11 \\
\hline 3 & $\mathrm{Cl}$ & $\mathrm{H}$ & - & $\mathrm{H}$ & $\mathrm{Me}$ & 6.11 & 6.02 & 0.09 & 5.79 & 0.32 & 6.05 & 0.06 & 6.00 & 0.11 \\
\hline 4 & $\mathrm{Br}$ & $\mathrm{H}$ & - & $\mathrm{H}$ & $\mathrm{Me}$ & 5.90 & 6.08 & -0.18 & $4.97^{c}$ & $0.93^{c}$ & 5.81 & 0.09 & $5.36^{c}$ & $0.54^{c}$ \\
\hline $5^{d}$ & $\mathrm{Cl}_{2}$ & $\mathrm{Cl}_{2}$ & - & $\mathrm{H}$ & $\mathrm{Me}$ & 5.94 & 5.97 & -0.03 & - & - & 5.80 & 0.14 & - & - \\
\hline 6 & $\mathrm{H}$ & $\mathrm{H}$ & - & $\mathrm{H}$ & $\mathrm{H}$ & 5.70 & 6.03 & -0.33 & 5.69 & 0.01 & 5.89 & -0.19 & 5.55 & 0.15 \\
\hline 7 & $\mathrm{Cl}$ & $\mathrm{H}$ & - & $\mathrm{H}$ & $\mathrm{Cl}$ & 5.23 & 5.29 & -0.06 & 5.37 & -0.14 & 5.44 & -0.21 & 5.62 & -0.39 \\
\hline 8 & $\mathrm{Br}$ & $\mathrm{H}$ & - & $\mathrm{H}$ & $\mathrm{Cl}$ & 4.73 & $5.86^{c}$ & $-1.13^{c}$ & 4.98 & -0.25 & $5.82^{c}$ & $-1.09^{c}$ & 4.95 & -0.22 \\
\hline 9 & $\mathrm{Cl}$ & $\mathrm{Cl}$ & $\mathrm{OMe}$ & $\mathrm{H}$ & $\mathrm{Me}$ & 4.62 & 4.41 & 0.21 & 4.71 & -0.09 & 4.59 & 0.03 & 4.73 & -0.11 \\
\hline 10 & $\mathrm{Cl}$ & $\mathrm{Cl}$ & $\mathrm{OMe}$ & $\mathrm{H}$ & $\mathrm{Cl}$ & 4.40 & 4.35 & 0.05 & 4.39 & 0.01 & 4.46 & -0.06 & 4.30 & 0.10 \\
\hline 11 & $\mathrm{Cl}$ & $\mathrm{Cl}$ & $\mathrm{OBu}$ & $\mathrm{H}$ & $\mathrm{Me}$ & 3.87 & 4.07 & -0.20 & 3.72 & 0.15 & 3.89 & -0.02 & 3.88 & -0.01 \\
\hline 12 & $\mathrm{H}$ & $\mathrm{H}$ & - & $\mathrm{Me}$ & $\mathrm{Me}$ & 4.23 & $6.02^{c}$ & $-1.79^{c}$ & $6.09^{c}$ & $-1.86^{c}$ & $5.96^{c}$ & $-1.73^{c}$ & $5.82^{c}$ & $-1.59^{c}$ \\
\hline \multirow[t]{2}{*}{13} & $\mathrm{DDC}^{e}$ & $\mathrm{H}$ & - & $\mathrm{H}$ & $\mathrm{Me}$ & 3.85 & 3.79 & 0.06 & 3.82 & 0.03 & 3.75 & 0.10 & 3.66 & 0.19 \\
\hline & Ave.$^{f}$ & & & & & & & 0.15 & & 0.12 & & 0.10 & & 0.15 \\
\hline
\end{tabular}

${ }^{a}$ predicted value. ${ }^{b}$ difference between observed values and predicted values. ${ }^{c}$ test set compounds. ${ }^{d}$ not aligned with template $\mathrm{C} .{ }^{e} 4,4-\mathrm{dimethyl}-2,6-$ dioxocyclohexyl. ${ }^{f}$ average residual of training set. 


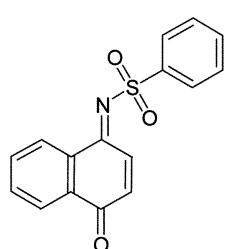

(A)

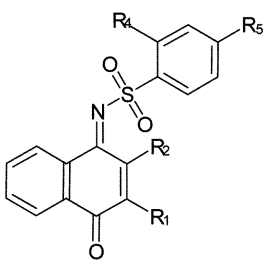

$(1-4,6-8,12,13)$

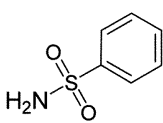

(B)



(C)
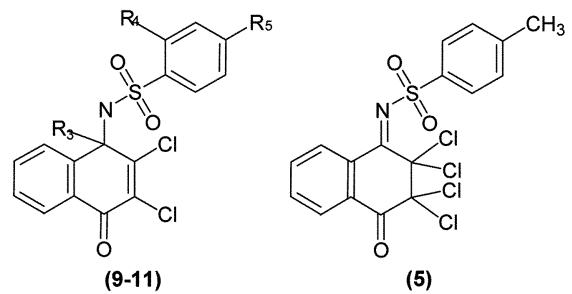

(5)
Figure 1. Structures of $N$-(4-oxo-1(4H)-naphthalenylidene)benzenesulfonamide derivatives (1-13) and compounds used as template (A-C) for molecular alignment.

lower number of components is better. Therefore, we put the number of components to 3 and derive the models. As the results of cross-validation, PRESS (predictive residual sum of squares), which is the squared prediction error between the observation value of test set molecules and their prediction, is also obtained.

The information obtained from the CoMFA and CoMSIA models is overlapped onto the $3 \mathrm{D}$ structure of $2\left(\mathrm{pI}_{50}=6.54\right)$ which shows the largest inhibition activities. To graphically analyze contribution from the steric and electrostatic fields, we put the CoMFA and CoMSIA coefficient into the contour map using field type (stdev*coeff.).

\section{Results and Discussion}

Inhibition and Molecular Template. The predicted values by CoMFA and CoMSIA model determined by the atom based fit alignment and the deviation between actual and predicted values were summarized in Table 1. Among the training set molecules, the most active compound is $\mathbf{2}$ with $\mathrm{R}_{1}=\mathrm{R}_{2}=\mathrm{Cl}, \mathrm{R}_{4}=\mathrm{H}, \mathrm{R}_{5}=$ methyl substituent, and its actual inhibition activity $\left(\mathrm{pI}_{50}\right)$ is 6.54 . Whereas the worst compound is $\mathbf{1 3}$ with $\mathrm{R}_{1}=4,4$-dimethyl-2,6-dioxocyclohexyl, $\mathrm{R}_{2}$ $=\mathrm{R}_{4}=\mathrm{H}, \mathrm{R}_{5}=$ methyl substituent, and its $\mathrm{pI}_{50}$ is 3.85 . Depending on the structural variation by the substituents at $\mathrm{R}_{1}-\mathrm{R}_{5}$, a relatively large the substituent effect $\left(\Delta \mathrm{pI}_{50}=2.69\right)$ was observed. As a pre-step to derive the models, we examine the most stable conformer of each molecule. The $\mathrm{R}_{1}$ and $\mathrm{R}_{2}$ groups on the 4-oxo-1 $(4 \mathrm{H})$ naphthalenylidene $(\mathrm{C})$ ring and, $R_{3}$ and $R_{4}$ groups on benzenesulfonamide (B) ring (Fig. 1) were minimized using the standard Tripos force field with the Gasteiger-Hückel charge ${ }^{13}$ and the simulated annealing method. ${ }^{12}$

The most stable conformers of the training set molecules, which have the lowest energy, are aligned in the three dimensional space by the atom based fit method. ${ }^{15}$ For example, Figure 2 shows the aligned structure using the benzenesulfonamide template (B). From Figure 2, we can

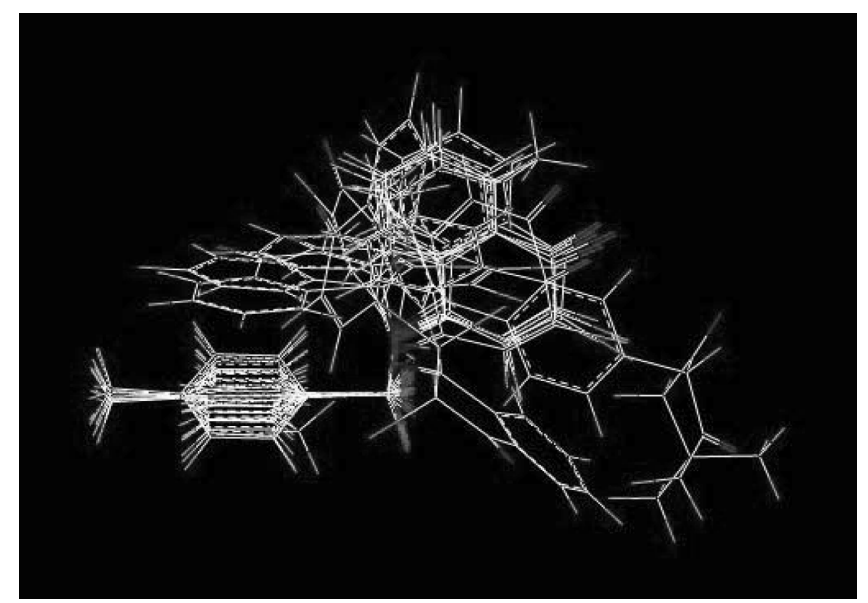

Figure 2. The superposition of the potential energy minimized substrate structures (training set) using atom based fit alignment with template B.

easily think that the inhibition activities vary depending on the three dimensional orientation of $\mathrm{R}_{1}-\mathrm{R}_{5}$ substituents, which are not included in the superposition of the ligands. Based on the PLS results, our models show good statistics in the order $(\mathrm{C})>(\mathrm{B})$ according to the change of templates. ${ }^{20}$

CoMFA Models for Inhibition. The statistical parameters of the $\mathrm{CoMFA}^{9}$ models with combination of various fields and the change of grid $(\AA)$ were summarized in Table 2 . The number of components of these models is 3 , and the grid $(\AA)$ is 2.5-3.0 $\AA$. In CoMFA, standard fields were used for describing the physicochemical properties of the molecules. As for the CoMFA Model 1 (template B), cross-validated $\mathrm{r}_{\text {cv. }}^{2}\left(\right.$ or $\left.\mathrm{q}^{2}\right)$ is 0.722 , non-cross-validated $\mathrm{r}^{2}$ ncv is 0.964 , and PRESS is 0.361 . The relative contributions of steric and electrostatic field were $45.7 \%$ and $54.3 \%$, respectively. The proportion of the two fields is almost equal. Figure 3 shows

Table 2. Summary of results from the best CoMFA and CoMSIA models

\begin{tabular}{|c|c|c|c|c|}
\hline \multirow{2}{*}{$\begin{array}{l}\text { 3D-QSAR } \\
\text { Models }\end{array}$} & \multicolumn{2}{|c|}{ CoMFA } & \multicolumn{2}{|c|}{ CoMSIA } \\
\hline & 1 & 2 & 1 & 2 \\
\hline Templates & B & $\mathrm{C}$ & B & $\mathrm{C}$ \\
\hline Fields & Stn & Stn & Ste $+E$ & Ste $+E$ \\
\hline Grid $(\AA)$ & 3.0 & 2.5 & 3.0 & 2.5 \\
\hline $\mathrm{NOC}^{a}$ & 3 & 3 & 3 & 3 \\
\hline $\operatorname{AF}(\alpha)^{b}$ & - & - & 0.3 & 0.3 \\
\hline$r_{\text {cv. }}^{2}\left(q^{2}\right)$ & 0.722 & 0.608 & 0.622 & 0.647 \\
\hline $\mathrm{r}_{\text {ncv. }}^{2}$ & 0.964 & 0.974 & 0.985 & 0.965 \\
\hline $\mathrm{SEE}^{c}$ & 0.227 & 0.199 & 0.145 & 0.230 \\
\hline $\mathrm{F}^{d}$ & 61.608 & 75.285 & 153.650 & 55.839 \\
\hline Steric & 0.457 & 0.668 & 0.369 & 0.342 \\
\hline Electrostatic & 0.543 & 0.332 & 0.631 & 0.658 \\
\hline PRESS $^{e}$ & 0.361 & 0.236 & 0.147 & 0.317 \\
\hline
\end{tabular}

Abbreviations: Stn; standard, Ste; steric \& E; electrostatic., ${ }^{a}$ number of component. ${ }^{b}$ attenuation factor. ${ }^{c}$ standard error estimate. ${ }^{d}$ fraction of explained versus unexplained variance. ${ }^{e}$ predictive residual sum of squares of the training set. 


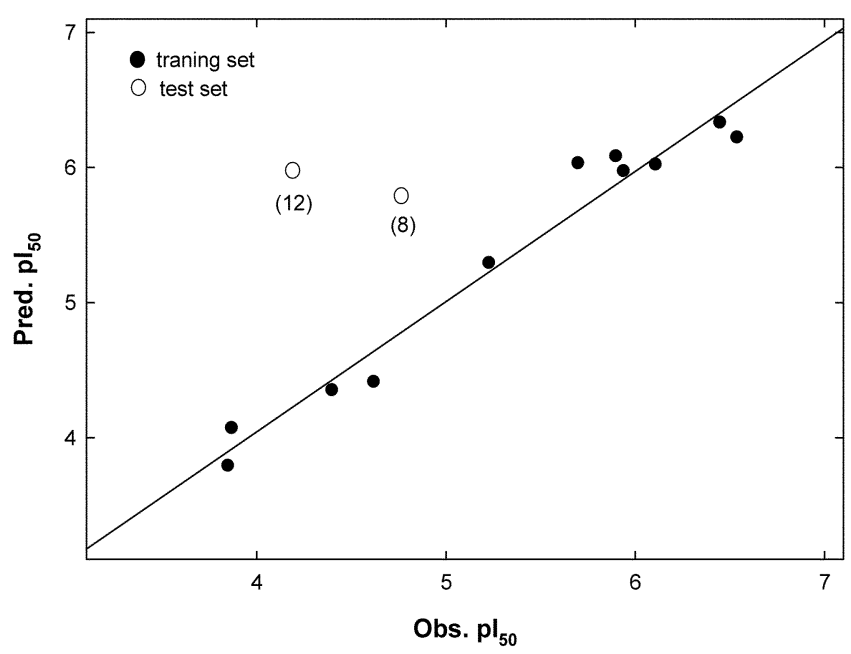

Figure 3. Relationships between observed inhibition values (obs.) and predicted values (pred.) by CoMFA model 1. (Training set: Pred. $\mathrm{pI}_{50}=0.963$ Obs.pI $\mathrm{I}_{50}+0.191, \mathrm{~s}=0.196, \mathrm{~F}=237.084, \mathrm{r}^{2}=$ $\left.0.963 \& \mathrm{q}^{2}=0.946\right)$

the relationship between the observed and the predicted values by this model. Although $\mathbf{8}$ and $\mathbf{1 2}$ show large deviations from the straight line, correlation coefficient of the training set is very good $\left(\mathrm{r}^{2}=0.963\right)$. When the clogP is added as an additional descriptor, ${ }^{16}$ the statistical results do not improve due to small contribution of hydrophobicity to the inhibition activities. This suggests that the substrate molecules already have enough hydrophobicity. ${ }^{21}$

As for CoMFA Model 2 (template C), cross-validated $r^{2}$. (or $\mathrm{q}^{2}$ ) is 0.608 , non-cross-validated $\mathrm{r}^{2}$ ncv is 0.974 and PRESS is 0.236 . The relative contributions of steric and electrostatic field were $66.8 \%$ and $33.2 \%$, respectively. The proportion of steric to electrostatic field is ca. $2: 1$, which means the steric field has twice as large an influence as the electrostatic field. As shown in Table 1, since the average



Figure 4. Variation of $\mathrm{q}^{2}$ upon changing the attenuation factors $(\alpha)$ used in the distance dependence between the probe atom and the atoms in the molecules with template $\mathrm{C}$ (The number on top \& bottom of the point indicates the optimum number of components). deviations between observed and predicted values by two models are similar, there are no big differences to explain the inhibition activities between templates.

CoMSIA Model for Inhibition. All the CoMSIA ${ }^{10}$ models are derived under the condition that the steric and electrostatic fields are combined. As for CoMSIA Model 1 (template B), cross-validated $r_{\text {cv. }}^{2}$ (or $\mathrm{q}^{2}$ ) is 0.622 , non-crossvalidated $r^{2}$ nc is 0.985 , PRESS is 0.147 . The relative contributions of steric and electrostatic field were $36.9 \%$ and $63.1 \%$, respectively. And as for CoMSIA Model 2 (template C), cross-validated $\mathrm{r}_{\text {cv. }}^{2}$ (or $\mathrm{q}^{2}$ ) is 0.647 , non-cross-validated $\mathrm{r}^{2}$ ncv is 0.965 , PRESS is 0.317 . The relative contributions of steric and electrostatic field were $34.2 \%$ and $65.8 \%$, respectively. In both cases, the contribution of the electrostatic field is twice larger than that for the steric field. Figure 4 shows the relationship between cross-validated $r_{c v}^{2}$. (or $\left.q^{2}\right)$ and attenuation factor $(\alpha)$ that is dependent upon the distance between the atoms in the molecules and the probe atoms. The best model which has the biggest $\mathrm{r}_{\mathrm{cv}}^{2}$ ( or $\left.\mathrm{q}^{2}\right)$ value is obtained when the number of components is 3 and $\alpha$ $=0.3$. The relationship between the observed and the predicted values by CoMSIA models shows the same tendency as that of CoMFA. In the CoMSIA analysis, Model 1 is significantly better than Model 2 judging from the average value of the deviation between the observed values and the predicted values by the two models. Therefore, template $\mathrm{B}$ is more reliable in explaining the inhibition activities. However, it is not useful to discuss the deviation for the test set molecules because the number of compounds is too small to derive any significant meaning.

CoMFA and CoMSIA Contour Maps. In order to visualize graphically the contribution of CoMFA and CoMSIA fields, we select the $2\left(\mathrm{pI}_{50}=6.54\right)$ as a representative case, which shows the largest inhibition activity. Then, we generated the contour maps showing steric fields and electrostatic fields according to the templates (B and C). For example, the one of the CoMFA contour maps is shown in Figure 5.

In the case of template $\mathrm{B}$, the sterically favored regions are

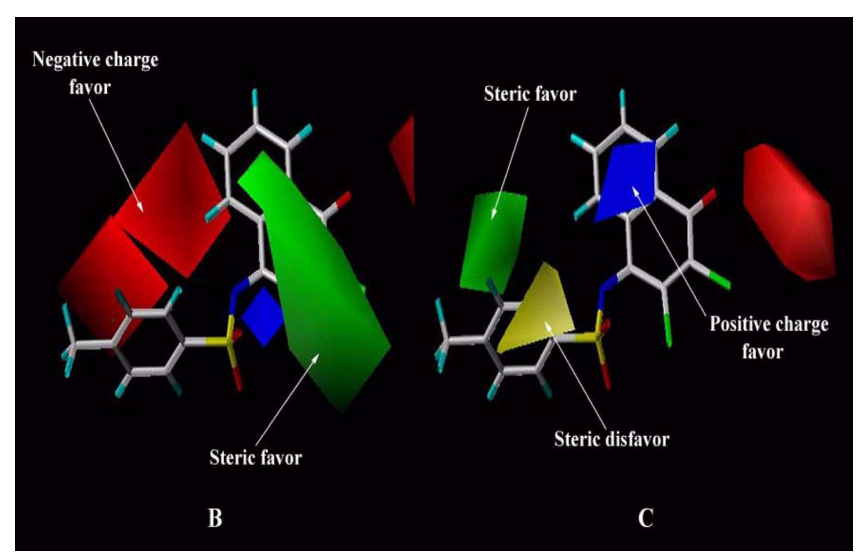

Figure 5. CoMFA contour maps for the steric and electrostatic field (stdev*coeff) on inhibition activity with template B and C. The most inhibition active compound (2) is shown in capped sticks. 
shown near $R_{1}$ and $R_{2}$ group - two parts over the wide range of 4-oxo-1(4H)-naphthalenylidene ring. And atoms $\mathrm{C}_{7}$ and $\mathrm{C}_{8}$ on the benzo group will have better inhibition activities if they have negative charge. In the case of template $\mathrm{C}$, however, the ortho position on the benzenesulfonyl ring is steric disfavored and the meta position is steric favored. It is expected that part of azomethine carbon atom will have increased inhibition activities if it has a positive charged atom. In addition, the oxygen atom of the carbonyl group will have better inhibition activities as it has a negative charge favor. The CoMSIA contour map shows a tendency similar to that of CoMFA.

By understanding the quantitative structure-activity relationship between the inhibition activities and quantitative structures of substrates molecules according to the results of the graphic analyses of the contour maps, it is expected that we can effectively use these results when designing new benzenesulfonamide derivatives with improved inhibition activities.

Acknowledgement. This work was supported by a grant (No. R11-2002-100-03005) from ERC program of the Korea Science and Engineering Foundation (KOSEF).

\section{References and Notes}

1. (a) Thomson, A. W. In Therapeutic Immunosuppression; Kluwer Academic Publishers: 2001; p 130. (b) Bohlin, L. Phytochem. Rev. 1995, 37, 137.

2. Pawson, T.; Scott, J. D. Science 1997, 278, 2075.

3. Shaw, K. T.; Ho, A. M.; Raghavan, A.; Kim, J.; Jain, J.; Park, J.; Sharma, S.; Rao, A.; Hogan, P. G. Proc. Natl. Acad. Sci., USA 1995, 92, 11205

4. Beals, C. R.; Clipstone, N. A.; Ho, S. N.; Crabtree, G. R. Genes. Dev. 1997, 11, 824 .

5. Aramburu, J.; Garcia-Cozar, F.; Raghavan, A.; Okamura, H.; Rao, A.; Hogan, P. G. Mol. Cell. 1998, 1, 627.

6. Aramburu, J.; Yaffe, M. B.; López-Rodríguez, C.; Cantley, L. C.; Hogan, P. G.; Rao, A. Science 1999, 285, 2129.

7. Roehrl, M. H.; Kang, S.; Aramburu, J.; Wagner, G.; Rao, A.;
Hogan, P. G. Proc. Natl. Acad. Sci. USA 2004, 101, 7544.

8. Kubinyi, H., In 3D-QSAR in Drug Design; Theory, Methods and Applications; Escom: Leiden, 1993.

9. Cramer, R. D.; Patterson, D. E.; Bunce, J. D. J. Am. Chem. Soc. 1988, 110, 5959.

10. (a) Klebe, G. Prospect Drug Discovery Des. 1998, 12, 87. (b) Klebe, G.; Abraham, U. J. Comput. Aid. Mol. Des. 1999, 13, 1. (c) Bohm, M.; Sturzebecher, J.; Klebe, G. J. Med. Chem. 1999, 42, 458.

11. Tripos Associates, Inc., 1699 S. Hanley Road, Suite 303, St. Louis, MO., 63144-2913 U.S.A., http://www.tripos.com/Bookshelf/ qsar/.

12. Kerr, R. Biophys. J. 1964, 67, 1501.

13. Purcell, W. P.; Singer, J. A. J. Chem. Eng. Data. 1967, 12, 235.

14. Klebe, G. In 3D QSAR Drug Design, Theory, Methods and Applications: Structural Alignment of Molecules; Kubinyi, H., Ed.; ESCOM: Leiden, 1993; pp 173-199.

15. Marshall, G. R.; Barry, C. D.; Bosshard, H. E.; Dammkoehler, R. A.; Dunn, D. A. In Computer-Assisted Drug Design: The Conformational Parameter in Drug Design; Active Analog Approach; Olsen, E. C.; Christoffersen, R. E., Eds.; American Chemical Society: Washington, D. C. 1979; pp 205-226.

16. Raichurkar, A. V.; Kulkarni, V. M. J. Med. Chem. 2003, 46, 4419.

17. Most of the compounds are composed of azomethine bond, but the compounds, 9-11 tend not to be aligned in template A, because they can rotate around the $\mathrm{C}-\mathrm{N}$ bonds axis. In addition, the $\mathrm{R}_{1}=\mathrm{R}_{2}=$ dichloro substituent, $\mathbf{5}$ is excluded, because the 4-oxo1(4H)-naphthalenylidene part is not aligned. In the case of template $\mathrm{B}$, on the other hand, though all the 13 compounds are aligned, the models of the compounds are derived with the $\mathbf{8}$ and 12 excluded. In the case of template $C$, the models are derived based on the subjects of all compounds that exclude the $\mathbf{4}$ and $\mathbf{1 2}$, as well as the $\mathbf{5}$.

18. (a) Stahle, L.; Wold, S. Prog. Med. Chem. 1988, 25, 291. (b) Geladi, P. J. Chemon. 1998, 2, 231.

19. Cramer, R. D., III.; Bunce, J. D.; Patterson, D. E. Quant. Struct. Act. Relat. 1988, 7, 18.

20. The models for all the substrate molecules $(n=13)$ derived from the combination of template, $(\mathrm{A})-(\mathrm{C})$ with various fields. All the derived models are statistically poor, but the correlation coefficient tends to be larger than the predictability $\left(r^{2}>q^{2}\right)$.

21. (a) Hansch, C.; Steward, A. R. J. Med. Chem. 1964, 7, 691. (b) Fujita, T. In Progress in Physical Organic Chemistry; Taft, R., Ed.; Wiely \& Sons: 1983; Vol. 14, pp 75-113. 